\title{
SELF-INTERSECTIONS OF SOME IMMERSED MANIFOLDS
}

\author{
BY
}

JOHN G. MILLER

The purpose of this paper is to prove a theorem, stated in $\S 3$, which describes the self-intersection manifolds of some immersions of differentiable manifolds in euclidean space in the metastable range. The description is in terms of $Z_{2}$-bordism, a concept introduced by Conner and Floyd [2] generalizing the cobordism theory of Thom [15]. In $\$ 4$ some consequences of the theorem are stated and in $\$ 5$ a method is developed for producing immersions of spheres whose self-intersections are the real projective spaces $P^{n}$ for all $n$.

This paper embodies the substance of a thesis submitted to Rice University, written with the advice of Professor Eldon Dyer.

During its preparation I was a guest at the City University of New York.

I am grateful to Professor John Milnor for his many suggestions.

1. All manifolds and maps will be differentiable of class $C^{\infty}$, and manifolds will be compact and without boundary unless otherwise stated. All homology will have $Z_{2}$ coefficients unless otherwise stated.

In addition to the standard results of differential topology, as found in Lang [5] or Milnor [9]-[12], some special material will be needed.

Two compact manifolds $N_{0}^{n}, N_{1}^{n}$ are (unoriented) cobordant if there exists a compact manifold with boundary $W^{n+1}$ such that $\partial W=N_{0} \cup N_{1}$. This can be shown to be an equivalence relation. The class of a manifold $M$ will be written $[M]$.

The disjoint union operation + and the cartesian product operation $\times$ make the set of all cobordism classes into a graded commutative $Z_{2}$-algebra $\mathfrak{R}$. The additive inverse of a cobordism class is itself, since $\partial(M \times I)=M \cup M$. The class of the empty manifold is the zero element. Thom [15] showed that $\mathfrak{R}$ is a polynomial algebra with one generator in each dimension not of the form $2^{j}-1$. The cobordism class of a manifold $N$ in $\mathfrak{N}$ is determined as follows: Let $w_{i}$ be the $i$ th Stiefel-Whitney class of the tangent bundle of $N$ [11] and [N] its fundamental homology class. Let $\pi$ be a sequence $\left(i_{1}, \ldots, i_{n}\right)$ such that $\sum_{j} j i_{j}=n$. The $\pi$ th Stiefel-Whitney number of $N$ is the value of the index $\left\langle w_{1}^{i_{1}} w_{2}^{i_{2}} \cdots w_{n}^{i_{n}},[N]\right\rangle$. Then two manifolds are cobordant iff these numbers are the same for all $\pi$.

These ideas received the following extension from Conner and Floyd [2]: The objects considered are manifolds with fixed-point free involutions, that is, diffeomorphisms $T$ such that $T^{2}=I$ and $T x \neq x$ for all $x$. These will be written $(M, T)$.

Received by the editors September 7, 1967. 
Two such, $\left(M_{0}^{m}, T_{0}\right),\left(M_{1}^{m}, T_{1}\right)$, are cobordant if there exists $\left(W^{m+1}, T\right)$ such that $\partial(W, T)=\left(M_{0}, T_{0}\right) \cup\left(M_{1}, T_{1}\right)$, the union of the given manifolds with their given involutions. Note that the quotient manifolds $M_{0} / T_{0}$ and $M_{1} / T_{1}$ are then cobordant in the original sense. Disjoint union, as above, equips the objects under this relation with the structure of a graded vector space over $Z_{2}$, denoted $\mathfrak{N}\left(B_{Z_{2}}\right)$. Further, $\mathfrak{R}\left(B_{Z_{2}}\right)$ is a module over $\mathfrak{R}$. If $[N] \in \mathfrak{R}$ and $[(M, T)] \in \mathfrak{R}\left(B_{Z_{2}}\right)$, then $(N \times M, 1 \times T)$ represents $[N][(M, T)]$, the involution $1 \times T$ acting in coordinate-wise fashion.

Conner and Floyd have shown that $\mathfrak{N}\left(B_{Z_{2}}\right)$ is a free module over $\mathfrak{N}$ with one generator in each dimension $\geqq 0$. These may be taken to be $\left(S^{n}, A^{n}\right)$, the standard sphere furnished with the antipodal map. In other words, any element may be expressed as a sum $[(M, T)]=\sum_{i=0}^{m}\left[V_{i}\right]\left[\left(S^{i}, A^{i}\right)\right]$, where $V_{i}$ is of dimension $m-i$.

These equivariant cobordism classes are determined by involution numbers analogous to the Stiefel-Whitney numbers described above. Given $\left(M^{m}, T\right)$, there is in $H^{1}(M / T)$ an element $c$, the characteristic class of the double covering $M \rightarrow M / T$. An involution number of $(M, T)$ is an integer $(\bmod 2)\left\langle w_{1}^{i_{1}} \cdots w_{m}^{i_{m}} c^{r},[M / T]\right\rangle$, where $\sum_{j} j i_{j}+r=m$, and the $w_{j}$ are the tangent Stiefel-Whitney classes of $M / T$. As above, two manifolds with involution are equivariantly cobordant iff all their involution numbers agree.

2. The immersions considered here will be of compact $n$-dimensional manifolds $N^{n}$ in $m$-dimensional euclidean space $R^{m}$ with the restriction of complete regularity.

DEFINITION. A collection of subspaces of a vector space intersect transversely if their orthogonal complements are linearly independent.

Let $T_{x}$ denote the tangent space at a point $x$ in a manifold.

Definition. An immersion $f: N^{n} \rightarrow R^{m}$ is completely regular if for $x_{1}, \ldots, x_{r}$ such that $f\left(x_{1}\right)=\cdots=f\left(x_{r}\right), f_{*} T_{x_{1}}, \ldots, f_{*} T_{x_{r}}$ intersect transversely.

If $3 n<2 m$, the case we will be interested in, complete regularity insures that $f$ has no triple points; and it follows that the set $\bar{I}_{f}=\{x \in N \mid \exists y, f(x)=f(y)\}$ is a compact $(2 n-m)$-dimensional submanifold. Then the image $I_{f}=f \bar{I}_{f}$ is a submanifold of $R^{m}$, which is doubly covered by $\bar{I}_{f}$ [7].

Let $f^{2}=f \times f \mid N \times N-\Delta(N): N \times N-\Delta(N) \rightarrow R^{m} \times R^{m}$, where $\Delta$ denotes the diagonal. Then $\bar{I}_{f}$ may be realized as $\left(f^{2}\right)^{-1} \Delta\left(R^{m}\right)$. The covering transformation $T_{I_{f}}$ of $\bar{I}_{f} \rightarrow I_{f}$ is given by the transposition $(x, y) \rightarrow(y, x)$.

Completely regular immersions possess two important properties. They are generic in the sense of [16]: Any immersion of a compact manifold and its derivatives up to any order may be approximated arbitrarily closely by a regularly homotopic completely regular immersion, and any immersion close enough to a completely regular immersion $g$, whose first two derivatives approximate those of $g$, is completely regular. Secondly, they are stable, in that under a sufficiently small regular homotopy the diffeomorphism type of the immersion, in a suitably defined sense, does not change. In particular, the intersection manifolds are diffeomorphic [8]. 
PROPOSITION. If $f$ and $g$ are regularly homotopic completely regular immersions $N^{n} \rightarrow R^{m}, 3 n<2 m$, then $\left(\bar{I}_{f}, T_{I_{f}}\right)$ and $\left(\bar{I}_{g}, T_{\bar{I}_{q}}\right)$ are cobordant .

According to Hirsch [4] there is an immersion $G: N \times I \rightarrow R^{m} \times I$ which is equal to $f \times$ id on $N \times[0,1 / 3]$ and $g \times$ id on $N \times[2 / 3,1]$. Now $f \times$ id and $g \times$ id are completely regular; for if, say, $f \times \operatorname{id}\left(x, t_{0}\right)=\left(f(x), t_{0}\right)=\left(f(y), t_{0}\right)=f \times \operatorname{id}\left(y, t_{0}\right)$, the orthogonal complements to the differentials of the tangent spaces in $R^{m} \times I$ are just the inclusions of those in $R^{m}$, which were assumed to be linearly independent.

Approximate $G$ by a completely regular immersion $H$ relative to $N \times[0,1 / 3]$ and $N \times[2 / 3,1]$. A calculation shows that $H$ has no triple points, so $\left(\bar{I}_{H}, T_{I_{H}}\right)$ is the required cobordism.

3. The intersection number of a completely regular immersion $f: N \rightarrow R^{2 n}$ is the number $(\bmod 2)$ of point self-intersections. Whitney [19] showed that for all $n$ there exist immersions with intersection number taking either value.

A manifold $N$ is said to be stably $k$-parallelizable, or briefly, $k \pi$, if for every complex $K$ of dimension $\leqq k$ and map $h: K \rightarrow N, k^{*} \nu$ is trivial, where $\nu$ is the stable normal bundle of $N$. This is equivalent to the condition that for some cell decomposition of $N$ the stable normal bundle restricted to the $k$-skeleton is trivial. If $k \geqq n$, then $N$ is called a $\pi$-manifold.

Given a formal power series $\alpha=\sum_{i} \alpha_{i} x^{i}$ over $Z_{2}$. It is known that $\alpha$ is a unit iff $\alpha_{0}=1$, and in that case its inverse may be formed by rational operations. Let $w_{N}$ denote the total tangent Stiefel-Whitney class of a manifold $N$.

THEOREM 1. Let $N^{n}$ be a $k \pi$-manifold, $k \geqq 2 n-m$, and $f: N \rightarrow R^{m}$ be a completely regular immersion with $3 n<2 m$. Let jf be the composition $N \stackrel{f}{\rightarrow} R^{m} \stackrel{j}{\rightarrow} R^{2 n}$, and c be the characteristic class of the covering $\bar{I}_{f} \rightarrow I_{f}$. Then $w_{I_{f}}=(1+c)^{n-m}=1 /(1+c)^{m-n}$. If the intersection number of $j$ is 0 , then $c^{2 n-m}=0$ and $\bar{I}_{f}$ equivariantly bounds. If the intersection number of $j$ is 1 , then $c^{2 n-m} \neq 0$ and $\bar{I}_{f}$ is equivariantly nonbounding.

Thus the cobordism type of $I_{f}$ and the equivariant cobordism type of $\bar{I}_{f}$ may be computed, as explained in $\S 1$, and depend only on $m, n$, and the intersection number of $j f$.

An analogue of this theorem can be proved by the same methods with the weaker hypothesis $4 n<3 m$, but the author prefers to avoid the necessary complications of statement and proof. Note that in the expansion $\left[\bar{I}_{f}\right]=\sum_{i=0}^{2 n-m}\left[M_{i}\right]\left[S^{i}, A^{i}\right]$ the only term which can have nonzero involution number $\left\langle c^{2 n-m},\left[M_{i} \times S^{i} / A^{i}\right]\right\rangle$ is $\left[S^{2 n-m}, A^{2 n-m}\right]$, since $M_{i} \times S^{i} / A^{i}=M_{i} \times P^{i}$, where $P^{i}$ is real projective $i$-space. Thus, if $\bar{I}_{f}$ does not equivariantly bound, the coefficient of $\left[S^{2 n-m}, A^{2 n-m}\right]$ is 1 . I have no examples of immersions for which any other terms are not zero, but nothing seems to preclude the possibility of their existence.

CoRollaRY 1. If $\left[\bar{I}_{f}\right] \neq 0$, then $\left[\bar{I}_{f}\right]=\left[S^{2 n-m}, A^{2 n-m}\right]$ iff $m>2 n-2^{s}$, where $2^{s}$ is the greatest power of 2 dividing $n+1$. 
This follows from comparing $w_{P^{2 n-m}}=(1+c)^{2 n-m+1}$ and $(1+c)^{n-m}$.

COROLlARY 2. If $n$ is even, then $\left[\bar{I}_{f}\right]=0$ for every completely regular immersion $f: N \rightarrow R^{m}$ with $m<2 n$.

Smale and Lashof [6] have shown that if in this case the intersection number of if is not zero, then the Euler class $\chi \in H^{n}(N ; Z)$ is not zero. But Hirsch [4] has shown that $\chi=0$ is a necessary and sufficient condition for an immersion of $N$ in $R^{2 n}$ to be regularly homotopic to one in $R^{2 n-1}$.

The proof of the theorem will be preceded by several lemmas. Let $k: R^{m} \rightarrow R^{m+1}$ be the standard inclusion.

LEMMA 1. Let $f: N^{n} \rightarrow R^{m}$ be a completely regular immersion, $3 n<2 m$, with $\bar{I}_{f}$ representing $\sum_{i=0}^{2 n-m}\left[M_{i}\right]\left[S^{i}, A^{i}\right]$. Then for any completely regular immersion $g$ regularly homotopic to $k f, \bar{I}_{g}$ represents $\sum_{i=1}^{2 n-m}\left[M_{i}\right]\left[S^{i-1}, A^{i-1}\right]$.

By the proposition it will suffice to find one such $g$. The proof will be divided into two parts.

Given a manifold with involution $\left(V^{v}, T\right)$, the line bundle $E_{V}$ associated to $T$ has total space the quotient of $V \times R$ by the relation $(x, s) \sim(T x,-s)$, base space $V / T$, and projection $p_{V}:(x, s) \rightarrow[x]$, where $[x]$ is the class of $x$ in $V / T$.

(1) Let $V^{v}$ be a manifold with involution $T$, representing $\sum_{i=0}^{v}\left[M_{i}\right]\left[S^{i}, A^{i}\right]$ $\in \mathfrak{R}\left(B_{Z_{2}}\right)$. Then if $r: V / T \rightarrow E_{V}$ is a section which is transverse to the zero section and $0_{r}$ is the set of zeros, then $\left(P_{V}^{-1} 0_{r}, T \mid P_{V}^{-1} 0_{r}\right)$ represents $\sum_{i=1}^{v}\left[M_{i}\right]\left[S^{i-1}, A^{i-1}\right]$.

By the hypotheses there exists an equivariant cobordism $(W, R)$ between $(V, T)$ and $\bigcup_{i=1}^{v}\left(M_{i} \times S^{i}, 1 \times A^{i}\right)=(X, A)$. I claim that there is a section $q_{i}$ of $E_{M_{i}} \times S^{i}$, transverse to the zero section and such that $0_{q_{i}}$ is $M_{i} \times S^{i-1} / T=M_{i} \times P^{i-1}$. Consider $S^{i}$ embedded as the unit sphere in $R^{i+1}$ and define $h(x), x \in S^{i}$, to be the $(i+1)$ th coordinate $x_{i+1}$ of $x$. Then define $\bar{q}_{i}(y, x)=(y, x, h(x)) \in M_{i} \times S^{i} \times R$. It is evident that $h\left(A^{i} x\right)=h(-x)=-h(x)$. Thus $\bar{q}_{i}(x)$ respects the identifications made in defining $E_{M_{i}} \times S^{i}$ and passes to a section $q_{i}: M_{i} \times S^{i} / A^{i}=M_{i} \times P^{i} \rightarrow E_{M_{i}} \times S^{i}$. Since $\partial h / \partial x_{i+1}$ is $\neq 0$ on $S^{i-1}, \bar{q}_{i}$ is transverse to the zero section and thus so is $q_{i}$. Define $q: X \rightarrow E_{X}$ to be the union of the $q_{i}$. Then $0_{q}=\bigcup_{i=1}^{n} M_{i} \times P^{i-1}$.

$X \mid A$ and $V / T$ have neighborhoods diffeomorphic to the products $(X \mid A) \times I$ and $(V / T) \times I$ in $W / R$. Define a section $v: W / R \rightarrow E_{W}$ to be the products $q \times$ id and $r \times$ id in these neighborhoods and transverse to the zero section elsewhere. Then $0_{v}$ is a manifold with boundary $0_{r} \cup 0_{q} .\left(p_{W}^{-1} 0_{v}, R \mid p_{W}^{-1} 0_{v}\right)$ is a manifold with involution, restricting on its boundary to $\left(p_{V}^{-1} 0_{r}, T \mid p_{V}^{-1} 0_{r}\right) \cup\left(p_{X}^{-1} 0_{q}, A \mid p_{X}^{-1} 0_{q}\right)$. But

$$
\begin{aligned}
\left(p_{\bar{X}}^{-1} 0_{q}, A \mid p_{X}^{-1} 0_{q}\right) & =\bigcup_{i=1}^{v}\left(M_{i} \times S^{i-1}, 1 \times A^{i} \mid M_{i} \times S^{i-1}\right) \\
& =\bigcup_{i=1}^{v}\left(M_{i} \times S^{i-1}, 1 \times A^{i-1}\right),
\end{aligned}
$$

which establishes (1). 
(2) Let $f$ be an immersion as above. Then if $r$ is any section of $E_{I_{f}}$ transverse to the zero section there is a completely regular immersion $g$ regularly homotopic to $k f$ such that $\bar{I}_{g}=\left(p_{I_{f}}^{-1} 0_{r}, T_{I_{f}} \mid p_{I_{f}}^{-1} 0_{r}\right)$.

$g$ will be constructed by first defining a map $m: \bar{I}_{f} \rightarrow I_{f} \times R \subset R^{m+1}$ and then extending $m$ to an immersion of $N$.

By the definition of $E_{I_{f}}$ there is a bundle map $s: \bar{I}_{f} \times R \rightarrow E_{I_{f}}$. Then there is the diagram

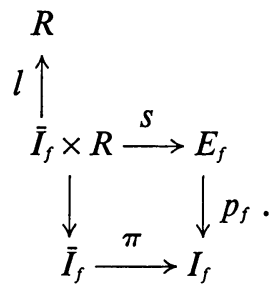

Let $\bar{r}$ be the section of $\bar{I}_{f}$ induced from $r$ by $\pi$. That is, $\bar{r}(x)=s^{-1} r \pi(x)$. Define $m(x)=(f(x), \operatorname{lr}(x))$. Then since $\operatorname{lr} x=-l \bar{r} T x$, by the identifications made in defining $E_{I_{f}}$, there exists $y$ such that $m(x)=m(y)$ iff $x \in \pi^{-1}\left(0_{r}\right)$. Further, $m\left(\bar{I}_{f}\right)$ meets $I_{f}$ transversely in $I_{f} \times R$, since $r$ meets the zero section in $E_{I_{f}}$ transversely and the bundle map $s$ is locally an isomorphism.

Let $U$ be a tubular $\varepsilon$-neighborhood of $\bar{I}_{f}$ in $N$ for small $\varepsilon>0$.

Let $d(x)$ be the distance of $x$ from $\bar{I}_{f}$ in $N$. Let $\rho$ be a real valued $C^{\infty}$ function defined on the reals satisfying

(i) $\rho(y)=1, y \leqq 0 ; \rho(y)=0, y \geqq \varepsilon$, and

(ii) $d \rho / d x \leqq 0[14]$.

Define $g$ as follows: If $x \notin U, g(x)=k f(x)$. If $w \in \bar{I}_{f}$ and $x$ is in the fiber of $w$ in $U$, $U_{w}, g(x)=[f(x), \rho(d(x)) \cdot \operatorname{lr}(w)] \in R^{m} \times R=R^{m+1}$. Then $g$ extends $m$, and its selfintersections are precisely those of $m$. Define a homotopy $H$ between $k f$ and $g$ by $H(x, t)=[f(x), 0]$ if $x \notin U$, and if $x \in U_{w}, H(x, t)=[f(x), \rho(d(x)) \cdot \operatorname{lr}(w) \cdot t]$.

Then $g$ is an immersion. This is clear if $x \notin U$. If $x \in U_{w}$, we may select a neighborhood of $w$ in $\bar{I}_{f}$ on which $m$ is an embedding. Then it is clear that $g$ embeds the restriction of $N$ to this neighborhood. Similarly $H(N, t)$ is an immersion for all $t$, so $H$ is a regular homotopy. Finally we must show that at any intersection point $z=f(x)=f(y)$ of $g$ the intersection is transverse.

In the case of an immersion like $g$ which has no triple points, this is easily seen to be equivalent to the condition that the images of the tangent spaces at $x$ and $y$ span that at $z$.

Now $z$ is also an intersection point of $f$. The projection $R^{m+1}=R^{m} \times R \rightarrow R^{m}$ carries $g$ into $f$, which was assumed to be completely regular, so the tangent spaces of the two leaves at $z$ span the subspace of the tangent space of $R^{m+1}$ orthogonal to the last coordinate. Since $g$ extends $m$ and the two leaves of $m\left(\bar{I}_{f}\right)$ at each intersection were shown to span the tangent space to $I_{f} \times R$, where $R$ is the last coordinate, the two tangent spaces at $z$ span the tangent space to $R^{m+1}$.

Combining (1) and (2) yields the lemma. 
Corollary. If $f$ is an immersion as above and $\bar{I}_{f}=\left(S^{2 n-m}, A^{2 n-m}\right)$, then we may choose $g$ so that $\bar{I}_{g}=\left(S^{2 n-m-1}, A^{2 n-m-1}\right)$.

In this case the construction of a section $q$ over $X / A$ made in (1) may be applied directly on $I_{f}$. (2) needs no modification.

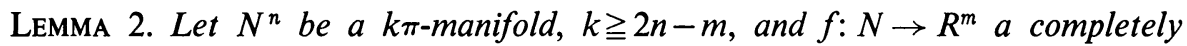
regular immersion with $3 n<2 m$. Let $\alpha$ be the line bundle over $I_{f}$ associated to $\pi: \bar{I}_{f} \rightarrow I_{f}, \varepsilon$ the trivial line bundle, and $c$ the characteristic class of $\alpha$. Then the normal bundle $\nu$ of $I_{f}$ in $R^{m}$ is $(m-n) \alpha \oplus(m-n) \varepsilon$ and thus its total Stiefel-Whitney class is $(1+c)^{m-n}$.

I claim that $\bar{I}_{f}$ is a $\pi$-manifold: it is diffeomorphic to $\left(f^{2}\right)^{-1} \Delta\left(R^{m}\right) \subset N \times M$. The normal bundle to $\left(f^{2}\right)^{-1} \Delta\left(R^{m}\right)$ in $N \times N$ is trivial, being induced from that of $\Delta\left(R^{m}\right)$ in $R^{m} \times R^{m}$. Now $\bar{I}_{f}$ is of dimension $2 n-m$, and being a differentiable manifold is homeomorphic to a complex [13]. Since $k \geqq 2 n-m$, it will suffice to show that $N \times N$ is a $k \pi$-manifold. Let $p_{1}, p_{2}$ be the projections of $N \times N$ on its factors. If $\eta$ is the stable normal bundle of $N$, the stable normal bundle of $N \times N$ may be written $p_{1}^{*} \eta \oplus p_{2}^{*} \eta$. If $N^{r}$ is the $r$-skeleton of $N$ in some cell decomposition, then under $p_{1}$, $N^{k} \times N$ maps onto $N^{k}$, so $p_{1}^{*} \eta \mid N^{k} \times N$ is trivial. Similarly $p_{2}^{*} \eta \mid N \times N^{k}$ is trivial. Thus $p_{1}^{*} \eta \oplus p_{2}^{*} \mid N^{k} \times N^{k}$ is trivial. But $(N \times N)^{k} \subset N^{k} \times N^{k}$.

$\bar{I}_{f}$ is contained in $N$ with normal bundle $\xi$ of dimension $n-(2 n-m)=m-n$. Since $N$ is a $k \pi$-manifold and $k \geqq 2 n-m$, $\xi$ is stably trivial. Since the fiber dimension $m-n$ is greater than the base dimension $2 n-m, \xi$ is trivial. Let $\left\{r_{i}\right\}, i=1, \ldots, m-n$, be a framing of $\xi$. If $f(x)=f(y)=z$, the normal space to $I_{f}$ at $z$ is the sum of the normal spaces to $\bar{I}_{f}$ in $N$ at $x$ and $y$, since the intersection is transverse. Each $r_{i}$ thus determines a two-dimensional subbundle $\nu_{i}$ of $\nu$. The $\nu_{i}$ are mutually isomorphic, for an automorphism of $\xi$ taking $r_{i}$ to $r_{j}$ induces an automorphism of $\nu$ taking $\nu_{i}$ to $v_{j}$.

Consider the trivial 2-plane bundle $\theta$ over $\bar{I}_{f}$ spanned by $r_{1}$ and a second vector field $s_{1}$. Define a bundle map $l: \theta \rightarrow \nu_{1}$ by $l r_{1}(x)=f_{*} r_{1}(x), l s_{1}(x)=f_{*} r_{1}(T x)$. The identifications made under this map are $a r_{1}(x)+b s_{1}(x) \sim a s_{1}(T x)+b r_{1}(T x)$. Let $q_{1}=r_{1}+s_{1}, q_{2}=r_{1}-s_{1}$. Then in terms of the $\left\{q_{i}\right\}$ the identifications are $c q_{1}(x)+d q_{2}(x)$ $\sim c q_{1}(T x)-d q_{2}(T x)$, so the image of the span of $q_{1}$ is trivial, and the image of that of $q_{2}$ is the line bundle associated to the covering $\bar{I}_{f} \rightarrow I_{f}$.

Proof of Theorem 1. It follows from Lemma 2 that the normal Stiefel-Whitney class of $I_{f}$ is $(1+c)^{m-n}$, where $c$ is truncated to some height $\leqq 2 n-m$. Thus by Whitney duality the tangent class is $1 /(1+c)^{m-n}$. Suppose that the intersection number of $j f$ is 0 . Then $c^{2 m-n}=0$, for if not $\bar{I}_{f}$ has the nonzero involution number $\left\langle c^{2 m-n},\left[I_{f}\right]\right\rangle$, and since $\left[S^{2 n-m}, A^{2 n-m}\right]$ is the only term with this involution number, it must have coefficient 1 in $\bar{I}_{f}$. By descending induction using Lemma 1 the intersection number of $j f$ is 1 , a contradiction. Then $\bar{I}_{f}$ has no nonzero involution numbers and is an equivariant boundary. 
If the intersection number of $j f$ is 1 we see using Lemma 1 that the coefficient of $\left[S^{2 n-m}, A^{2 n-m}\right]$ is 1 , and so $\left\langle c^{2 n-m},\left[I_{f}\right]\right\rangle \neq 0$, and $\bar{I}_{f}$ does not equivariantly bound.

4. Robert Wells [17] has shown that if $3 n<2 m, k \geqq 2 n-m$, and $N^{n}$ is a $k \pi$ manifold, then every manifold-with-involution occurring as the self-intersection of a completely regular immersion $N \rightarrow R^{m}$ may be realized as that of some completely regular immersion of the sphere $S^{n} \rightarrow R^{m}$. Thus according to Theorem 1 the existence of immersions of $k \pi$-manifolds in the above range with equivariantly nonbounding self-intersections is equivalent to the existence of immersions $S^{n} \rightarrow R^{m}$ which go under the inclusion $R^{m} \rightarrow R^{2 n}$ into immersions with intersection number $1(\bmod 2)$.

Recall Smale's theory of immersions of spheres in euclidean space [14]. If $n<m$, the group of immersions of $S^{n}$ in $R^{m}$ under the operation of connected sum is isomorphic to $\pi_{n}\left(V_{m, n}\right)$, where $V_{m, n}$ is the Stiefel manifold of orthogonal $n$-frames in $m$-space. Further, the inclusion of immersions $S^{n} \rightarrow R^{m} \rightarrow R^{m+1}$ corresponds to the homomorphism $\pi_{n}\left(V_{m, n}\right) \rightarrow \pi_{n}\left(V_{m+1, n}\right)$ induced from the map $V_{m, n} \rightarrow V_{m+1, n}$. It was remarked in $\S 3$ that self-intersections are uninteresting for $n$ even. We thus confine attention to odd $n$. In this case Smale's construction shows that the immersion of $S^{n}$ in $R^{2 n}$ with intersection number 1 corresponds to the nonzero element of $\pi_{n}\left(V_{2 n, n}\right) \approx Z_{2}$. We thus have

TheOREM 2. Consider $n, m$ with $3 n<2 m$ and $n$ odd. Then there exists an $n$-dimensional $k \pi$-manifold, $k \geqq 2 n-m$, completely regularly immersed in $R^{m}$ with equivariantly nonbounding self-intersection iff the homomorphism $\pi_{n}\left(V_{m, n}\right) \rightarrow \pi_{n}\left(V_{2 n, n}\right)$ is not zero. In fact the manifold may always be taken to be a sphere.

5. In many cases the question of the existence of self-intersections may be settled geometrically, answering the homotopy-theoretic question of the last section indirectly.

Throughout this section $P^{r}$ will denote real projective space and $S^{r}$ the sphere considered as a covering space of $P^{r}$. I will consider immersions $f: P^{n} \rightarrow R^{m}$ with $3 n+1<2 m$. The method used here to find examples of self-intersections consists essentially of considering maps of projective spaces, passing to the associated maps of spheres given by the covering projection, and putting these into general position.

These examples are special cases of those found independently by Robert Wells [17]. Wells has also contributed to the simplification of my hypotheses.

Let $\alpha_{r}$ be the canonical line bundle over $P^{r}$, and $c_{r}$ the order of $\alpha_{r}$ in $\tilde{K} \tilde{O}\left(P^{r}\right)$ (the reduced $K O$ group).

THEOREM 3. Given $n$ and $m, 3 n+1<2 m$. Then if $n+1 \cong 0\left(\bmod c_{2 n-m}\right)$ there exists a completely regular immersion $S^{n} \rightarrow R^{m+1}$ with self-intersection $P^{2 n-m-1}$.

Since by Adams' calculations [1] $c_{r}$ is finite, such immersions exist for all values of $2 n-m$ for an infinite number of $m$. 
The principal fact needed is as follows:

Lemma 3. Given $n$ and $m, 3 n<2 m$ such that the stable normal bundle of $P^{n}$ restricted to $P^{2 n-m}$ is trivial. Then there is a map $g: S^{n} \rightarrow R^{m}$ whose singular set in $S^{n}$ has two components with the following structure: One component is a $(2 n-m)$ sphere with image $P^{2 n-m}$, of which a neighborhood is mapped by a completely regular immersion. The other is a $(2 n-m)$-manifold containing the singular points.

Consider any map $P^{n} \rightarrow R^{m}$. Haefliger [3] shows that in the stated dimensions it may be approximated by a map $f$ whose singular set, $Y_{f}$, is a submanifold of dimension $2 n-m$.

Since $2 n-m+2 n-m<n$ there is by general position an isotopy of $P^{2 n-m}$ in $P^{n}$ separating $P^{2 n-m}$ from $Y_{f}$. By the isotopy extension theorem [12], this isotopy extends to an isotopy $H: P^{n} \times I \rightarrow P^{n}$ such that for $f H_{1}: P^{n} \rightarrow R^{m}, f H_{1}\left(P^{2 n-m}\right)$ $\cap Y_{f H_{1}}=\varnothing$. I will assume this to be the case, writing $f$ for $f H_{1}$.

Let $P^{n}-Y_{f}$ have the Riemannian metric $d$ induced from that of $R^{m}$ by $f$ and let $K$ be a tubular $\varepsilon$-neighborhood of $P^{2 n-m}$ in $P^{n}$, with $\varepsilon$ chosen so that $K \cap Y_{f}=\varnothing$. Then since if in the given range the normal bundle of $P^{n}$ restricted to $P^{2 n-m}$ is stably trivial it is trivial, and $K$ contracts onto $P^{2 n-m}$, we may choose a framing $\left\{r_{i}\right\}, i=0, \ldots, m-n-1$, of the normal bundle of $P^{n}$ restricted to $K$.

$P^{r}$ is covered by $r+1$ coordinate neighborhoods $\left\{V_{i}\right\}$, where $V_{i}$ is the region where the $i$ th homogeneous coordinate is nonzero. $V_{i}$ lifts to $S^{r}$ as the union of the two open hemispheres $U_{i}^{+}$and $U_{i}^{-}$. Let $\delta_{i}(x)=1$ if $x \in U_{i}^{+},=-1$ if $x \in U_{i}^{-}$, and $=0$ if $x \notin U_{i}^{+}$or $U_{i}^{-}$.

$P^{2 n-m} \subset P^{n}$ may be considered as the intersection of $m-n$ copies of $P^{n-1},\left\{P_{i}^{n-1}\right\}$, where $P_{i}^{n-1}$ is the set of zeros of the $i$ th homogeneous coordinate.

Consider the normal disc bundle to $P^{n}$ as a submanifold of $R^{m}$. Let $p: S^{n} \rightarrow P^{n}$ be the projection. Define a map $g^{\prime}: p^{-1} K \rightarrow R^{m}$ by

$$
g^{\prime}(x)=\sum_{i=0}^{m-n-1} \delta_{i}(x) d\left(p x, P_{i}^{n-1}\right) r_{i}(f p x) .
$$

Let $g: S^{n} \rightarrow R^{m}$ be a map agreeing with $g^{\prime}$ on $p^{-1} K$ and whose singular set is a $(2 n-m)$-dimensional manifold, which exists by the theorem of Haefliger cited above.

I will show that $g \mid p^{-1} K$ is a completely regular immersion with $I_{g}=P^{2 n-m}$. To show that $g \mid p^{-1} K$ is an immersion it will suffice that $\delta_{i}(x) d\left(p x, P_{i}^{n-1}\right)$ be a $C^{\infty}$ function of $x$, for then $g$ will give a local section over $f K$, since $p$ is a local diffeomorphism, and thus $g$ will be locally an embedding.

If $x \in U_{i}^{+}, \quad \delta_{i}(x) d\left(p x, P_{i}^{n-1}\right)=d\left(p x, P_{i}^{n-1}\right)$. If $x \in U_{i}^{-}, \quad \delta_{i}(x) d\left(p x, P_{i}^{n-1}\right)=$ $-d\left(p x, P_{i}^{n-1}\right)$, and $d\left(p x, P_{i}^{n-1}\right)$ is $C^{\infty}$ on $p^{-1} K \cap U_{i}^{ \pm}$. Thus there remains only the case $x \notin U_{i}^{+}, U_{i}^{-}$. Let $S_{i}^{n-1}=S^{n}-\left(U_{i}^{+} \cup U_{i}^{-}\right)$. Since $p$ is locally a diffeomorphism, in a small neighborhood $X$ of $x$ we may write $\delta_{i}(x) d\left(p x, P_{i}^{n-1}\right)=\delta_{i}(x) \bar{d}\left(x, S_{i}^{n-1}\right)$, where $\bar{d}$ is the metric on $Z$ induced from $d$ by $p$. Let $x_{n}$ be the normal coordinate of 
$S_{i}^{n-1}$ in $S^{n}$, the positive direction being into $U_{i}^{+}$. Then locally $\delta_{i}(x) \bar{d}\left(x, S_{i}^{n-1}\right)$ $= \pm x_{n}$.

If $x_{1}, x_{2} \in p^{-1} x, x \in K-P^{2 n-m}$, then $\delta_{i}\left(x_{1}\right) \neq \delta_{i}\left(x_{2}\right)$ for some $i, 0 \leqq i<m-n$. Thus the self-intersection set of $g \mid p^{-1} K$ is $P^{2 n-m}$.

We must show that the intersection is transverse. To do this it will suffice to show that at every intersection point $x=f p\left(x_{1}\right)=f p\left(x_{2}\right)$ the leaves containing $x_{1}$ and $x_{2}$ have distinct derivatives with respect to $m-n$ independent directions normal to $f P^{2 n-m}$ in $f P^{n}$. Let $W$ be a small neighborhood of $x$ in $f P^{n}$ contained in $f K$. Let $W_{1}$ and $W_{2}$ be the components of $p^{-1} f^{-1} W$ which contain $x_{1}$ and $x_{2}$.

Define a partial coordinate system in $W$ by letting $w_{i}$ be the normal coordinate to $f P_{i}^{n-1}$ in $f P^{n}$ with positive direction chosen so that $w_{i}(f p y)>0$ if $y \in W_{1} \cap U_{i}^{+}$, and thus $w_{i}(f p y)<0$ if $y \in W_{2} \cap U_{i}^{+}$. Then the $w_{i}$ are independent functions on $W$, since the $P_{i}^{n-1}$ intersect transversely in $P^{n}$, and they lift under $p^{-1} f^{-1}$ to give partial coordinate systems $\left\{w_{i}^{1}\right\},\left\{w_{i}^{2}\right\}$. Let $g_{i}=g \mid w_{i}$. Since $p f$ is a diffeomorphism on each of $W_{1}, W_{2}$, we may write $g_{1}$ and $g_{2}$ in terms of the coordinates $\left\{w_{i}\right\}$ instead of $\left\{w_{i}^{1}\right\},\left\{w_{i}^{2}\right\}$. Then

$$
\left.\left.\frac{\partial g_{j}}{\partial w_{i}}\right]_{x}=\frac{\partial}{\partial w_{i}}\right]_{x} \sum_{k=0}^{m-n-1} \delta_{k}\left(x_{j}\right) d\left(f p x_{j}, f P_{i}^{n-1}\right) r_{k}\left(f p x_{j}\right)
$$

Rewriting as above, this is equal to

$$
\left.\frac{\partial}{\partial w_{i}}\right]_{x}(-1)^{j+1} \sum_{k=0}^{m-n-1} w_{k}\left(f p x_{j}\right) r_{k}\left(f p x_{j}\right)=(-1)^{j+1} r_{i}(x) .
$$

Since $r_{i}(x) \neq 0, \quad \partial g_{1} / \partial w_{i} \neq \partial g_{2} / \partial w_{i}$ for $0 \leqq i<m-n-1$, and the intersection is transverse.

Proof of Theorem 3. The normal bundle of $P^{2 n-m}$ splits as the sum of the normal bundle of $P^{2 n-m}$ in $P^{n}$ and the stable normal bundle of $P^{n}$ restricted to $P^{2 n-m}$. The former is the $(m-n)$-fold sum of $\alpha$. The tangent bundle of $P^{2 n-m}$ is stably the $(2 n-m+1)$-fold sum of $\alpha$ [10]. Thus, to apply Lemma 3 we must have that the stable normal bundle of $P^{2 n-m}$ is $(m-n) \alpha$. Since the stable normal bundle is uniquely characterized by the property that its sum with the stable tangent bundle is trivial, it will suffice that $(m-n)+(2 n-m+1)=n+1 \cong 0\left(\bmod c_{2 n-m}\right)$. Then a map $g$ exists as in Lemma 3 . The following is easily established.

Lemma 4. Let $A^{a}$ and $B^{b}$ be disjoint submanifolds of $S^{n}$ such that $a+b+1<n$. Then $A$ and $B$ are separated by an $(n-1)$-sphere embedded in $S^{n}$.

Thus, since $2 n-m+2 n-m+1<n, p^{-1} P^{2 n-m}$ and $Y_{g}-p^{-1} P^{2 n-m}$ may be separated by an $(n-1)$-sphere in $S^{n}$, which is then embedded by $g$. So under the hypotheses of Lemma 3 there exists a disc $D^{n}$ immersed in $R^{m}$ with $P^{2 n-m}$ as selfintersection and which is embedded in a neighborhood $V$ of $\partial D^{n}$.

Taking the composition $D^{n} \stackrel{g}{\rightarrow} R^{m} \rightarrow R^{m+1}$ we may attach a cone $C$ over $\partial D^{n}$, forming a sphere $S^{n}$ which is (topologically) embedded on $C \cup V$. Now by a 
theorem of Haefliger [3], since $3 n<2 m$, this embedding may be approximated by a differentiable embedding of $C \cup V$, leaving $g$ unaltered outside of $V$.

Now we may apply the corollary to Lemma 1 to produce an immersion $S^{n} \rightarrow R^{m+1}$ with the desired self-intersection.

\section{REFERENCES}

1. J. F. Adams, Vector fields on spheres, Ann. of Math. 75 (1962), 602-632.

2. P. Conner and E. Floyd, Differentiable periodic maps, Academic Press, New York, 1964.

3. A. Haefliger, Plongements différentiables de variétés dans variétés, Comment. Math. Helv. 36 (1961), 57-82.

4. M. Hirsch, Immersions of manifolds, Trans. Amer. Math. Soc. 93 (1959), 242-276.

5. S. Lang, Introduction to differentiable manifolds, Interscience, New York, 1962.

6. R. Lashof and S. Smale, On the immersion of manifolds in euclidean space, Ann. of Math. 68 (1958), 562-583.

7. —_, Self-intersections of immersed manifolds, J. Math. Mech. 8 (1959), 143-157.

8. H. I. Levine, Singularities of differentiable mappings. I, from lectures of R. Thom, Bonn, 1959.

9. J. Milnor, Differential topology, Princeton Univ., Princeton, N. J., 1958.

10. - Lectures on characteristic classes, Princeton Univ., Princeton, N. J., 1957.

11. - The Sard-Brown theorem and elementary topology, Princeton Univ., Princeton, N. J., 1964.

12. — Differentiable structures, Princeton Univ., Princeton, N. J., 1961.

13. J. Munkres, Elementary differential topology, Princeton Univ. Press, Princeton, N. J., 1963.

14. S. Smale, The classification of immersions of spheres in euclidean space, Ann. of Math. 69 (1957), 327-344.

15. R. Thom, Quelques propriétés globales des variétés différentiables, Comment. Math. Helv. 28 (1954), 17-86.

16. — Les singularités des applications différentiables, Ann. Inst. Fourier (Grenoble) 6 (1955), 43-87.

17. R. Wells, Modification of intersections (to appear).

18. H. Whitney, The self-intersections of a smooth n-manifold in $2 n$-space, Ann. of Math. 45 (1944), 220-245.

The City University OF New York, NeW YoRK, NeW YoRK

The University of California, Los Angeles, California 